\title{
Impulsive Control of a Chaotic Financial System
}

\section{Yong Wang}

Department of Information Science and Technology, Tianjin University of Finance and Economics, Tianjin, China

ywang@tjufe.edu.cn

Keywords: impulsive control, finance system, stability, chaos

\begin{abstract}
The impulsive control of a finance system is investigated in this paper, and the finance system can be asymptotically controlled to the origin by using impulsive control. Based on the new comparison theorem of impulsive system, this paper obtains some sufficient for the stabilization of the finance system via impulsive control with varying impulsive intervals, A numerical simulation illustrates the effectiveness of the proposed result.
\end{abstract}

\section{Introduction}

The chaotic financial system is a complex nonlinear system, which has some interesting phenomena, such as the limit cycle, stable periodic orbits, quasi periodic attractor, and chaotic attractor. After the system enters the chaotic state, the stable periodic orbit turns into an unstable periodic orbit, and how to turn the chaotic motion of the system into a regular motion is the purpose of chaos control. The pulse control systems have been considered by a number of authors [1-4]. The recent, impulse control is also widely used to control chaotic systems [5-7].

Gao qin et al.[8] formulated a financial model, studied the chaos and bifurcations problems of this financial model, and got some good results. In this paper, we study the stability of this chaotic financial model by using the pulse control method, and obtain the sufficient conditions for asymptotic stability of the financial system. The results show that some beneficial adjustments can be made to the operation of the market economy.

\section{The pulse stability of the financial model}

Gao qin et al.[8] discussed a financial model, shown as following.

$$
\left\{\begin{array}{l}
x(t)=z(t)+(y(t)-a) x(t), \\
y(t)=1-b y(t)-x^{2}(t), \\
z \dot{(t)}=-x(t)-c z(t),
\end{array}\right.
$$

Where $a \geq 0$ is the saving amount, $\mathrm{b} \geq 0$ is the cost per investment, and $c \geq 0$ is the elasticity of demand of commercial markets. Chaos is found through numerical simulation (Fig. 1).

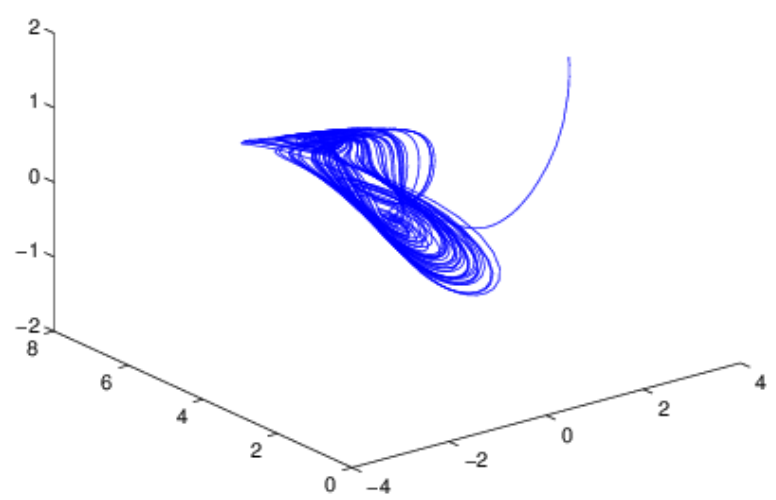

Fig. 1 chaotic attractor: $a=4, b=0.1, c=1$. 
Let $\left(x^{*}, y^{*}, z^{*}\right)$ is a steady-state of the system (1) and $X_{1}=x-x^{*}, X_{2}=y-y^{*}, X_{3}=z-z^{*}$, $X^{T}=\left(X_{1}, X_{2}, X_{2}\right)$, the system (1) can become

$$
\dot{X}=A X+\Phi(X) \text {. }
$$

Where $A=\left(\begin{array}{ccc}y^{*}-a & x^{*} & 1 \\ -2 x^{*} & -b & 0 \\ -1 & 0 & -c ?\end{array}\right) \quad \Phi(X)=\left(\begin{array}{c}X_{1} X_{2} \\ -X_{1}^{2} \\ 0 ?\end{array}\right)$

We add impulsive control to the system (2)

$$
\left\{\begin{array}{lc}
\dot{X}=A X+\Phi(X) & t \neq t_{k} \\
\left.\Delta X\right|_{t=t_{k}}=I(k, X)=B X & t=t_{k}, k=1,2,3, \cdots
\end{array}\right.
$$

Where $\left\{t_{k}: k=1,2,3, \cdots\right\}$ satisfy $\Delta_{1}=\sup _{0 \leq j \leq \infty}\left\{t_{2 j+1}-t_{2 j}\right\}<\infty, \quad \Delta_{2}=\sup _{0 \leq j \leq \infty}\left\{t_{2 j}-t_{2 j-1}\right\}<\infty$ and $t_{2 j+1}-t_{2 j} \leq \varepsilon\left(t_{2 j}-t_{2 j-1}\right), \varepsilon$ is constant.

Theorem 1. Suppose that $n \times n$ matrix $\mathrm{P}$ is symmetric and positive definite, $\lambda_{1}>0, \lambda_{2}>0$ is the maximum and minimum eigenvalue, respectively. Let $Q=P A+A^{T} P$ where $A^{T}$ is transpose of $\mathrm{A}, \mathrm{Q}$ is maximum characteristic value of $P^{-1} Q$, d is maximum characteristic value of $P^{1}\left(I+B^{T}\right) P(I+B)$. And if there is a $\xi>1$ and non-increment function $K(t) \geq m>0$, which is differentiable at $t \neq t_{k}$ satisfies

$$
-\frac{D^{+} K(t)}{K(t)} \leq q+2 M \sqrt{\frac{\lambda_{2}}{\lambda_{1}}} \leq \frac{1}{(1+\varepsilon) \Delta_{2}} \ln \frac{K\left(t_{2 k}^{+}\right) K\left(t_{2 k-1}^{+}\right)}{K\left(t_{2 k+1}\right) K\left(t_{2 k}\right) \xi d^{2}}
$$

Or

$$
-\frac{D^{+} K(t)}{K(t)} \leq q+2 M \sqrt{\frac{\lambda_{2}}{\lambda_{1}}} \leq \frac{1}{\max \left\{\Delta_{1}, \Delta_{2}\right\}} \ln \frac{K\left(t_{k}^{+}\right)}{K\left(t_{k+1}\right) \xi d^{2}}
$$

Then the pulse system (3) is asymptotically stable, where $M=\max \left|X_{1}\right|$. (Particularly, when $P=I, \lambda_{1}=\lambda_{2}=1$ )

Proof: Suppose $V(t, X)=X^{T} P X, t \neq t_{i}, P \neq I$, we have

$$
K(t) D^{+} V(t, X)+D^{+} K(t) V(t, X)=K(t)\left[X^{T}\left(A^{T} P+P A\right) X+X^{T} P \Phi(X)+\Phi^{T}(X) P X\right]+\dot{K}(t) V(t, X)
$$$$
\leq K(t)\left[X^{T} Q X+2 \sqrt{X^{T} P X} \Phi(X) \sqrt{\Phi^{T}(X) P \Phi(X)}\right]+\dot{K}(t) V(t, X)
$$$$
\leq K(t)\left[q X^{T} P X+2 \sqrt{\lambda_{2}} \sqrt{X^{T} P X} \sqrt{\Phi^{T}(X) \Phi(X)}\right]+\dot{K}(t) V(t, X)
$$$$
\leq K(t)\left[q X^{T} P X+2 \sqrt{\lambda_{2}} \sqrt{X^{T} P X} \sqrt{X_{1}^{2}\left(X_{1}^{2}+X_{2}^{2}+X_{3}^{2}\right)}\right]+\dot{K}(t) V(t, X)
$$$$
\leq K(t)\left[q X^{T} P X+2 \frac{1}{\sqrt{P}} \sqrt{X_{1}^{2}} \sqrt{\lambda_{2}} \sqrt{X^{T} P X} \sqrt{X^{T} P X}\right]+\dot{K}(t) V(t, X)
$$$$
\leq\left[q+2 M \sqrt{\frac{\lambda_{2}}{\lambda_{1}}}+\frac{\dot{K(t)}}{K(t)}\right] K(t) V(t, X)
$$

And

$$
\begin{aligned}
& K\left(t_{k}+0\right) V\left(t_{k}+0, X+B X\right) \leq K\left(t_{k}\right)(X+B X)^{T} P(X+B X) \\
& =K\left(t_{k}\right) X^{T}(I+B)^{T} P(I+B) \leq d K\left(t_{k}\right) V\left(t_{k}, X\right)
\end{aligned}
$$

Suppose that $g(t, \omega)=\left[q+2 M \sqrt{\frac{\lambda_{2}}{\lambda_{1}}}+\frac{K(t)}{K(t)}\right] \omega, \Phi_{k}=d \omega$, then the conditions (1-3) in the lemma 1 [14] are satisfied. By the lemma 1, the stability of pulse system (3) is contained in the following comparison system. 


$$
\left\{\begin{array}{l}
\omega(t)=\left[q+2 M \sqrt{\lambda_{2} / \lambda_{1}}+\dot{K(t) / K(t)}\right] \omega(t), \quad t \neq t_{k} \\
\omega\left(t_{k}^{0}\right)=d \omega\left(t_{k}\right) \\
\omega\left(t_{k}^{0}\right)=\omega_{0} \geq 0
\end{array}\right.
$$

Now let's consider the conditions in lemma 2 [9], because

$$
\begin{aligned}
& \sup _{k}\left\{\operatorname{dexp}\left[q+2 M \sqrt{\frac{\lambda_{2}}{\lambda_{1}}}\left(t_{k+1}-t_{k}\right)+\ln \frac{K\left(t_{k+1}\right)}{K\left(t_{k}\right)}\right]\right\} \\
& \leq \operatorname{dexp}\left(q+2 M \sqrt{\frac{\lambda_{2}}{\lambda_{1}}}\right)\left(\max \left\{\Delta_{1}, \Delta_{2}\right\}<\infty\right.
\end{aligned}
$$

Then, the condition (2) in the lemma 2 [9] are satisfied.

$$
\begin{aligned}
& \left(q+2 M \sqrt{\frac{\lambda_{2}}{\lambda_{1}}}\right)\left(t_{2 k+1}-t_{2 k-1}\right)+\ln \frac{K\left(t_{2 k+1}\right) K\left(t_{2 k}\right)}{K\left(t_{2 k}^{+}\right) K\left(t_{2 k-1}^{+}\right)} \\
& =\left(q+2 M \sqrt{\frac{\lambda_{2}}{\lambda_{1}}}\right)\left(t_{2 k+1}-t_{2 k}+t_{2 k}-t_{2 k-1}\right)+\ln \frac{K\left(t_{2 k+1}\right) K\left(t_{2 k}\right)}{K\left(t_{2 k}^{+}\right) K\left(t_{2 k-1}^{+}\right)} \\
& \leq\left(q+2 M \sqrt{\frac{\lambda_{2}}{\lambda_{1}}}\right)\left(\Delta_{1}+\Delta_{2}\right)+\ln \frac{K\left(t_{2 k+1}\right) K\left(t_{2 k}\right)}{K\left(t_{2 k}^{+}\right) K\left(t_{2 k-1}^{+}\right)} \\
& \leq\left(q+2 M \sqrt{\frac{\lambda_{2}}{\lambda_{1}}}\right)(1+\varepsilon) \Delta_{2}+\ln \frac{K\left(t_{2 k+1}\right) K\left(t_{2 k}\right)}{K\left(t_{2 k}^{+}\right) K\left(t_{2 k-1}^{+}\right)} \leq-\ln \left(\xi d^{2}\right)
\end{aligned}
$$

Or

$$
\left(q+2 M \sqrt{\frac{\lambda_{2}}{\lambda_{1}}}\right)\left(t_{k+1}-t_{2 k}\right)+\ln \frac{K\left(t_{k+1}\right)}{K\left(t_{i}^{+}\right)} \leq\left(q+2 M \sqrt{\frac{\lambda_{2}}{\lambda_{1}}}\right) \max \left\{\Delta_{1}, \Delta_{2}\right\}+\ln \frac{K\left(t_{k+1}\right)}{K\left(t_{i}^{+}\right)} \leq-\ln (\xi d)
$$

Hence, the condition (3) in the lemma 2 [9] are also satisfied. Then the pulse system (3) is asymptotically stable.

Theorem 2. If

$$
\lim _{T \rightarrow \infty} \frac{i(t, t+T)}{T}=p=\frac{1}{\tau}
$$

Where $i(t, t+T)$ is the number of $t_{i}$ in the $[t, t+T]$.

And

$$
0 \leq q+2 M \sqrt{\frac{\lambda_{2}}{\lambda_{1}}} \leq-p \ln (\xi d), \xi>1
$$

Then the trivial solution of the system (3) is asymptotical stable.

Proof: From the previous hypothesis, we have

$$
g(t, \omega)=\left[q+2 M \sqrt{\frac{\lambda_{2}}{\lambda_{1}}}+\frac{K(t)}{K(t)}\right] \omega(t)=\dot{\lambda}(t)
$$

Hence

$$
\int_{t_{k}}^{t_{k+1}} \dot{\lambda}(t) d t=\left(q+2 M \sqrt{\frac{\lambda_{2}}{\lambda_{1}}}\right)\left(t_{k+1}-t_{k}\right)+\ln \frac{K\left(t_{k+1}\right)}{K\left(t_{k}\right)}
$$

And $K(t)$ is non-increasing function, so $\ln \frac{K\left(t_{k+1}\right)}{K\left(t_{k}\right)} \leq 0$, hence

$$
\int_{t_{k}}^{t_{k+1}} \dot{\lambda}(t) d t \leq\left(q+2 M \sqrt{\frac{\lambda_{2}}{\lambda_{1}}}\right)\left(t_{k+1}-t_{k}\right)
$$

By using equation (2), we have 


$$
\int_{t_{k}}^{t_{k+1}}\left(q+2 M \sqrt{\frac{\lambda_{2}}{\lambda_{1}}}\right) d t+\ln (\xi d) \leq 0, \xi>1 \quad \text { for all } k .
$$

Hence

$$
\int_{t_{k}}^{t_{k+1}} \dot{\lambda}(t) d t \leq \ln (\xi d)
$$

By the lemma 2 [9], the trivial solution of the system (3) is asymptotical stable.

\section{Numerical Simulation}

For the system (1), when $a=4, b=0.1, c=1$, chaos is found through numerical simulation (Fig. 1).

Take the equilibrium point $P_{0}\left(0, \frac{1}{b}, 0\right)$ of the system (1), then

$$
A=\left(\begin{array}{ccc}
6 & 0 & 1 \\
0 & -0.1 & 0 \\
-1 & 0 & -1
\end{array}\right) \quad A+A^{T}=\left(\begin{array}{ccc}
12 & 0 & 0 \\
0 & -0.2 & 0 \\
0 & 0 & -2
\end{array}\right)
$$

For the sake of simplicity, Assuming $P=I$, then $q=\max (12,-0.2,-2)=12$, supposing $M=2$, taking

$$
B=\left(\begin{array}{ccc}
k_{1} & 0 & 0 \\
0 & k_{2} & 0 \\
0 & 0 & k_{3}
\end{array}\right)=\left(\begin{array}{ccc}
-0.58 & 0 & 0 \\
0 & -0.65 & 0 \\
0 & 0 & -0.75
\end{array}\right)
$$

We have $k=\max \left(k_{1}, k_{2}, k_{3}\right)=-0.58$, and using the Theorem 2 , then

$$
0 \leq \tau=\frac{1}{p} \leq-\ln \left(\xi(1+k)^{2} / q=0.029, \xi=2.3\right.
$$

The result of numerical simulation is as shown in Fig. 2.

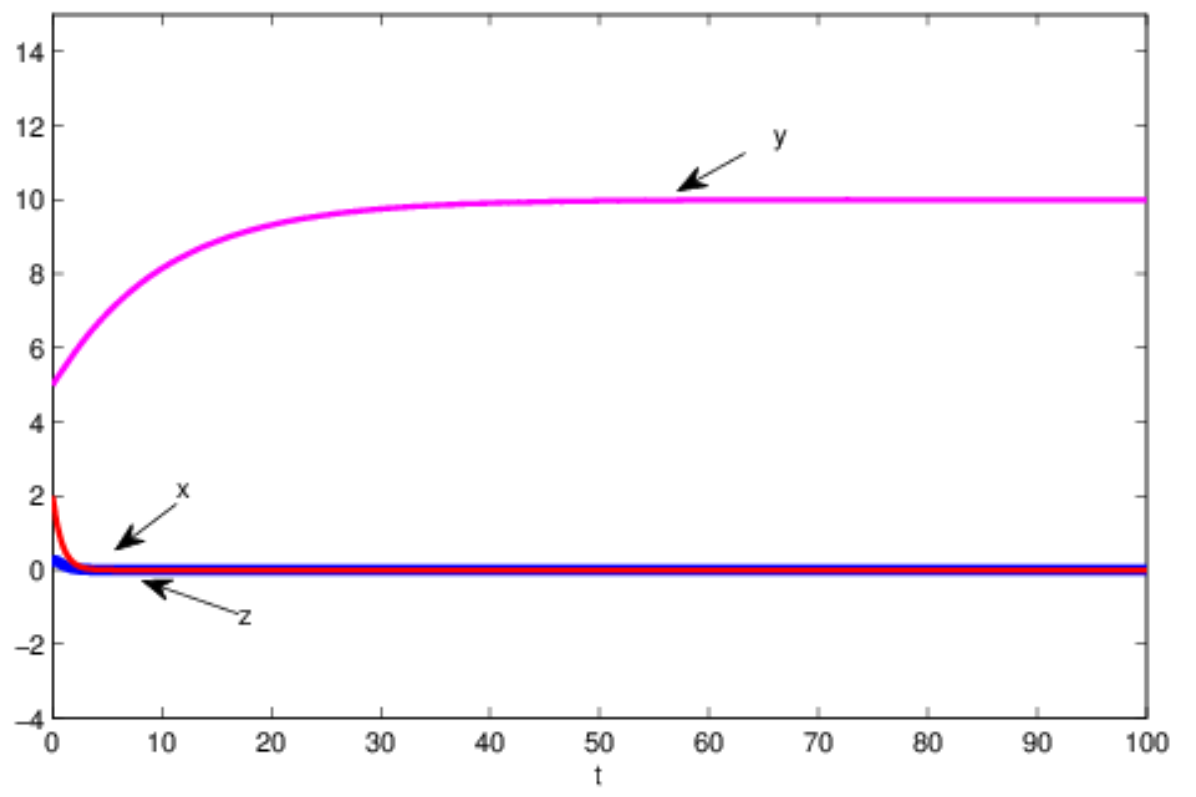

Fig. 2 When $a=4, b=0.1, c=1$, the equilibrium $P_{0}\left(0, \frac{1}{b}, 0\right)$ of system (1) is asymptotically stable.

\section{Conclusion}

The impulsive control of a finance system is investigated in this paper, and the finance system can be asymptotically controlled to the equilibrium point by using impulsive control. This paper obtains 
some sufficient for the stabilization of the finance system via impulsive control with varying impulsive intervals. This method can control chaotic motion effectively, can turn chaotic irregular motion into regular motion, and achieve the purpose of chaos control. In the actual commercial market, through our control, to some extent, we can effectively regulate the rapid and healthy development of economy.

\section{Acknowledgements}

The authors wish to express their gratitude to the editors and the reviewers for the helpful comments. This work is supported in part by NNSF of China (No. 11701410).

\section{References}

[1] L.N. Qian, Q.S. Lu, Dynamical behaviors of a prey-predator system with impulsive control, J. Math. Anal. Appl. 363 (2010) 345-356.

[2] Jitao Sun, Impulsive control of a financial model, Physics Letters A . 335 (2005) 282-288.

[3] T. Yang, Impulsive control, IEEE Trans, Autom. Control. 44(5) (1999) 1081-1083.

[4] R.Q. Shi, L.S. Chen, An impulsive predator-prey model with disease in the prey for integrated pest management, Commun Nonlinear Sci Numer Simulat. 15 (2010) 421-429.

[5] H.L. Xu, K.L. Teo, Stabilizability of discrete chaotic systems via unified impulsive control, Physics Letters A. 2 (2009) 235-240.

[6] X.H. Zhang, Anmar. Khadra, Impulsive stability of chaotic systems represented by T-S model, Chaos, Solitons and Fractals. 41(2009)1863-1869.

[7] Fen, Mehmet Onur, and M. Akhmet, Impulsive SICNNs with chaotic postsynaptic currents, Discrete and Continuous Dynamical Systems - Series B. 21 (2017) 1119-1148.

[8] Q. Gao, J.H. Ma, Chaos and Hopf bifurcation of a finance system, Nonlinear Dyn. 58 (2009) 209-216.

[9] J.T. Sun, Y.P. Zhang, Q.D. Wu, Less conservative conditions for asymptotic stability of impulsive control systems, IEEE Trans. Automatic Contr. 48(5) (2003) 829-831. 